\title{
Diagnostic Relevance of Overexpressed Serine Threonine Tyrosine Kinase/Novel Oncogene with Kinase Domain (STYK1/ NOK) mRNA in Colorectal Cancer
}

\author{
Ayla Valinezhad Orang ${ }^{1}$, Reza Safaralizadeh ${ }^{1 *}$, Mohammad Ali Hosseinpour \\ Feizi $^{1}$, Mohammad Hossein Somi ${ }^{2}$
}

\begin{abstract}
Background: Alterations in gene expression levels or mutations of tyrosine kinases are detected in some human cancers. In this study, we examined whether serine threonine tyrosine kinase 1 (STYK1)/novel oncogene with kinase domain (NOK) is overexpressed in patients with colorectal cancer. We also examined the clinical relevance of STYK1/NOK expression in cancer tissues. Materials and Methods: In tumor samples of patients with colorectal cancer and their matched non-cancerous samples, STYK1/NOK messenger RNA (mRNA) expression was analyzed by quantitative reverse transcriptase polymerase chain reaction. Associations between the expression levels of STYK1/NOK and clinicopathological characteristics of colorectal cancer were also assessed using Mann-Whitney U and Kruskal-Wallis tests. Results: Upregulation of STYK1/NOK was found in cancer tissues even at early stage of colorectal cancer compared to normal adjacent tissues. The optimal cutoff point of 0.198 the STYK1/NOK expression showed 0.78 sensitivity and 0.75 specificity for diagnosis. Overexpressed STYK1/NOK was correlated with tumor size but had no association with other clinicopathological characteristics of colorectal cancer. Conclusions: These results indicate that STYK1/NOK mRNA is widely expressed in the patients with colorectal cancer and suggest that inhibition of this molecule could potentially serve as a novel therapeutic target.
\end{abstract}

Keywords: Colorectal cancer - oncogene - tyrosine kinase - NOK/STYK1 - diagnosis

Asian Pac J Cancer Prev, 15 (16), 6685-6689

\section{Introduction}

Colorectal cancer (CRC) is the fourth most common cancer in the world and the most common form of colorectal cancer presentation is primary adenocarcinoma with non-urgent symptoms with non-urgent symptoms (Safaee et al., 2010, Siegel et al., 2013). Given the effectiveness of early diagnosis and treatment in colorectal cancer and the performance gaps of current non-invasive colorectal cancer markers, the development of novel biomarkers could result in improved patient outcomes for colorectal cancer (Barrett et al., 2006, Shemirani et al., 2011). Tyrosine kinases are of the former class that their abnormalities cause them to stuck in "on" position and contribute to the uncontrolled proliferation and anticancer drug resistance of a vast spectrum of human cancer cells (Hubbard and Till, 2000). In the light of In the light of these evidences and reports, kinase inhibition is of the considerable interest in recent research work (Zwick et al., 2002). Despite the gradual progress in colorectal cancer prognosis, the detection of cancer in early stages remains a relatively open research field (Samanian et al., 2011,
Barouni et al., 2012, Karimi et al., 2013). To increase the efficiency of cancer management programs, it is of urgent importance to identify candidate genes that their unique signature may hold promise as a novel diagnostic tool for detecting tumors at an early stage of colorectal cancer, particularly adenocarcinomas.

Receptor protein tyrosine kinases (RPTKs) are currently well-recognized for their pivotal roles in diverse cellular processes (Blume-Jensen and Hunter, 2001). Their dysregulation has been reported to result in an abnormal kinase activity and malignant transformation (Shepard et al., 2008). The structure of these subfamilies consists of a diverse ligand-specific ectodomain, a single transmembrane domain and an intracellular tyrosine kinase domain (Fambrough et al., 1999).

Serine Threonine Tyrosine kinase (STYK1)/ Novel oncogene kinase (NOK) is a newly identified member of RPTK-like protein family that lacks extracellular domain enabling it to act in a non-specific ligand manner (Liu et al., 2004). It is reported to be an important factor in tumorigenesis and metastasis in nude mice, and it is and it is proved to lead growth factor-independent cell 
proliferation among murine, bone-marrow-derived, lymphatic $\mathrm{BaF} 3$ cells and surface-adhesion-independent growth and colony forming in NIH3T3 and BaF3 cells (Chen et al., 2005, Li et al., 2009, Ding et al., 2012). Furthermore, NOK/STYK1 oncogenic potential in breast, lung, prostate and ovarian cancers has been reported (Amachika et al., 2007, Kimbro et al., 2008, Jackson et al., 2009). It is therefore an intriguing possibility that STYK1/NOK serves as a critical molecule in colorectal tumorigenesis and is thus of immediate interest as a candidate in the colorectal cancer prognosis and treatment. In this paper, we hypnotized that over-expression of STYK1/NOK may be an inevitable circumstance in cancer cells. However, expression level of this gene has not been yet quantified in colorectal cancer cells and the previous observations raised the question that to what extent the CRC tissue samples express STYK1/NOK compared to normal tissue samples.

In line with previous studies, we quantitatively analyzed the expression levels of STYK1/NOK in CRC tissues relative to their non-tumor counterparts. Moreover, the potential relationship between STYK1/NOK levels and clinicopathological and prognostic outcomes in CRC patients has been investigated.

\section{Materials and Methods}

\section{Patients and Tissue samples}

In this prospective study, a total of 40 CRC samples and normal adjacent tissues were collected following colonoscopy and sigmoidoscopy at Imam Reza Hospital (Tabriz, Iran), the first affiliated hospital of Tabriz University of Medical Sciences. The non-tumor counterparts were obtained from a section of the resected specimen at the farthest distance from tumor $(>2 \mathrm{~cm}$ from tumor). All study participants were Iranian-born individuals. The study was approved by the Research Ethics Committee of Imam Reza Hospital in accordance with institutional protocol and informed consents were obtained from all patients. Resected specimens were routinely processed for histopathological assessment. The clinicopathological factors and histological grades of cancer were classified using the TNM staging system of the American Joint Committee on Cancer (AJCC; 2010) and International Union Against Cancer (UICC), according to the standard of the World Health Organization (WHO). A number of cases were excluded from subsequent statistical analysis for the following reasons: the patients had a previous or secondary malignancy and/ or had undergone chemotherapy, radiation therapy or immunotherapy, the samples had histology other than adenocarcinomas.

\section{Sample preparation and RNA isolation}

All the tissue samples were immediately flash frozen in liquid nitrogen and stored at $-80^{\circ} \mathrm{C}$ until RNA extraction. We applied phenol based total RNA extraction using TRIzol reagent (Takara) according to the manufacturer's instructions with some small changes. Briefly, tissue samples were homogenized quickly along with liquid nitrogen and then suspended in $1 \mathrm{ml}$ TRIzol reagent and incubated at room temperature for $15 \mathrm{~min}$. By adding
$300 \mu$ chloroform (MERK) and then centrifugation at $12,000 \times \mathrm{g}$ for $15 \mathrm{~min}$ at $4^{\circ} \mathrm{C}$ nucleic acids were recovered from the lysate. The aqueous phase was carefully transferred into another microcentrifuge tube. Isopropanol was used to precipitate nucleic acid and stored at $20^{\circ} \mathrm{C}$ overnight followed by a $13,000 \times \mathrm{g}$ centrifuge for $10 \mathrm{~min}$ at $4^{\circ} \mathrm{C}$, pelleting the nucleic acid. The pellet was washed with $75 \%$ ethanol, air dried and at the end RNA was dissolved in diethyl pyrocarbonate (DEPC)-treated water. The RNA concentration and purity were measured with a NanoDrop ND-1000 Spectrophotometer. The integrity of the RNAs was checked using agarose gel electrophoresis stained with an intercalating dye. The extracted RNAs were stored at $-80^{\circ} \mathrm{C}$ until cDNA synthesis. To degrade any DNA contamination in extracted RNAs we performed a $10 \mu l$ DNase I treatment reaction which contained $1 \mu \mathrm{g}$ of total RNA, $1 \mu \mathrm{l} 10 \mathrm{X}$ reaction buffer, $\mathrm{I} \mu \mathrm{l}$ of $1 \mathrm{U} / \mu \mathrm{l}$ of DNase I (Takara) and DEPC-treated water and was incubated at $37^{\circ} \mathrm{C}$ for $15 \mathrm{~min}$. To heat inactive the DNase I, $1 \mu \mathrm{l}$ of 25 $\mathrm{nM}$ Ethylenediaminetetraacetic acid (EDTA) was added to the previous mixture and (was) firstly incubated at 65 $\mathrm{C}$ for $15 \mathrm{~min}$ and then was replaced on ice for $1 \mathrm{~min}$ with the Eppendorf 5331 thermal cycler.

\section{Reverse transcription reaction}

The reverse transcription to synthetize cDNA was conducted using RevertAid ${ }^{\mathrm{TM}}$ First Strand cDNA Synthesis Kit (Fermentas) and was divided into two parts. First reaction was a $12 \mu \mathrm{l}$ mixture containing $8 \mu \mathrm{l}$ of total RNA, was procured from the DNase I treatment reaction, $1 \mu \mathrm{l}$ oligo(dt) primer, DEPC-treated water, and was incubated at $65^{\circ} \mathrm{C}$ for $5 \mathrm{~min}$ in thermal cycler. The second one was a reverse transcription reaction added to the previous nuclease-free tube of fist reaction and contained $4 \mu \mathrm{l} 5 \mathrm{X}$ reaction buffer, $1 \mu \mathrm{l}$ RiboLock RNase Inhibitor $(20 \mathrm{u} / \mu \mathrm{l}), 2 \mu \mathrm{l}$ of $10 \mathrm{nM}$ dNTP Mix, $1 \mu \mathrm{l}$ RevertAid M-MulV Reverse Transcriptase $(200 \mathrm{u} / \mu \mathrm{l})$ and DEPCtreated water. The total $20 \mu \mathrm{l}$ mixture was firstly incubated at $37^{\circ} \mathrm{C}$ for $15 \mathrm{~min}$, followed by incubation at $85^{\circ} \mathrm{C}$ for $5 \mathrm{~min}$ for reverse transcriptase deactivation. The first stranded synthetized tube was restored at $-20^{\circ} \mathrm{C}$ until following procedure.

\section{Real-time polymerase chain reaction}

STYK1/NOK mRNA expression was determined by real time polymerase chain reaction by using a Power SYBR Green (Takara) with an iCycler $\mathrm{iQ}^{\mathrm{TM}}$ (Biorad). Results were normalized to the expression of glyceraldehyde-3-phosphate dehydrogenase (GAPDH). The gene-specific primers for STYK1/NOK and GAPDH were as follow:

STYK1/NOK forward primer, 5'-TCTCGGGA AATCTGAAATGATGC-3'; reverse primer: 5'-GGACT GGGACAGCAGAAGTG-3';

GAPDH forward primer, 5'-GTGAACCATG AGAAGTATGACAAC-3'; Reverse primer: 5'-CATGAGTCCTTCCACGATACC-3'.

The $20 \mu \mathrm{l}$ mixture of PCR consisted of $10 \mu \mathrm{l} \mathrm{SYBR}$ Green suppermix, DEPC-treated water, $0.5 \mu$ l forward primer, $0.5 \mu \mathrm{l}$ reverse primer and $1-3 \mu \mathrm{l}$ reverse transcribed product. 
The cycling program involved preliminary denaturation at $95^{\circ} \mathrm{C}$ for $5 \mathrm{~min}$, followed by 45 cycles of denaturation at $95^{\circ} \mathrm{C}$ for $15 \mathrm{~s}$, annealing at $60^{\circ} \mathrm{C}$ for $30 \mathrm{~s}$ and elongation at $72^{\circ} \mathrm{C}$ for $30 \mathrm{~s}$, followed by a final elongation step at $60^{\circ} \mathrm{C}$ for $10 \mathrm{~min}$. All samples were processed in triplicate. The threshold cycle (CT) was defined as the cycle number at which the fluorescence passed the fixed threshold. A control without a template was included in each experiment. The final products of real-time PCR were confirmed by Polyacrylamide gel electrophoresis (PAGE).

\section{Statistical analysis}

The expression levels of STYK1/NOK in CRC tissues relative to matched non-tumor counterparts were analyzed using the $2^{-\Delta \Delta \mathrm{Ct}}$ method. Briefly, the threshold cycle $(\mathrm{Ct})$ of fluorescence for each sample was determined. $\Delta \mathrm{Ct}$ indicated the difference in expression levels with the $\mathrm{Ct}$ value between STYK1/NOK and GAPDH $\left(\Delta \mathrm{Ct}=\mathrm{Ct}_{\text {STYK1/ }}\right.$ NOK $\left.-\mathrm{Ct}_{\mathrm{GAPDH}}\right)$, and $\Delta \Delta \mathrm{Ct}$ indicated the difference in the $\Delta \mathrm{Ct}$ value between cancer tissue and the matched control $\left(\Delta \Delta \mathrm{Ct}=\Delta \mathrm{Ct}_{\text {cancer }}-\Delta \mathrm{Ct}_{\text {control }}\right)$. The $2-^{\Delta \Delta \mathrm{Ct}}$ value (fold value) was also calculated. When the fold value was $>1$, there was a high expression of STYK1/NOK in the cancer tissues compared to their non tumorous counterparts. The statistical differences in STYK1/NOK expression in cancer tissues relative to the matched adjacent non tumor tissues were analyzed by a paired t-test. Moreover, the association between STYK1/NOK expression and clinicopathological parameters was analyzed by a non parametric test (Mann Whitney U test between 2 groups and Kruskal Wallis $\mathrm{H}$ test for $\geq 3$ groups). Statistical analysis was performed using the GraphPad Prism 6. Moreover, Receiver operating characteristic (ROC) curves were constructed to evaluate the specificity and sensitivity of predicting CRC and normal tissue by STYK1/NOK and the sensitivity/specificity at various cutoff values was calculated using SigmaPlot.

\section{Results}

Expression of STYK1/NOK in CRC tissues.

Quantitative real-time reverse transcription-polymerase chain reaction (qRT-PCR) analysis of STYK1/NOK was performed in 36 pairs of CRC tissues and matched adjacent non-tumor counterparts. The results showed that STYK/NOK expression levels were significantly elevated in CRC tissues compared to normal matches. The value of $\Delta \mathrm{Ct}($ mean $\pm \mathrm{SD})$ was $0.037 \pm 0.030$ in CRC tissues and $0.013 \pm 0.011$ in their matching adjacent nontumor tissues. The mean difference and the range of SD was $0.024 \pm 0.0051$ (p value $<0.01$, paired t-test; Figure 1).

Capability of STYK1/NOK to function as a CRC tumor marker. Receiver operating characteristic (ROC) curves was constructed and the area under the curve (AROC) was calculated to evaluate the specificity and sensitivity of predicting CRC and normal tissue by STYK1/NOK expression levels. Based on the analysis of ROC curved STYK1/NOK mRNA showed a ROC area (AROC) of 0.79 . (Figure. $2 ; \mathrm{p}<0.001$ ) The plot shows sensitivity and specificity at different cut-off points. To calculate the optimal cut-off value we performed a post-

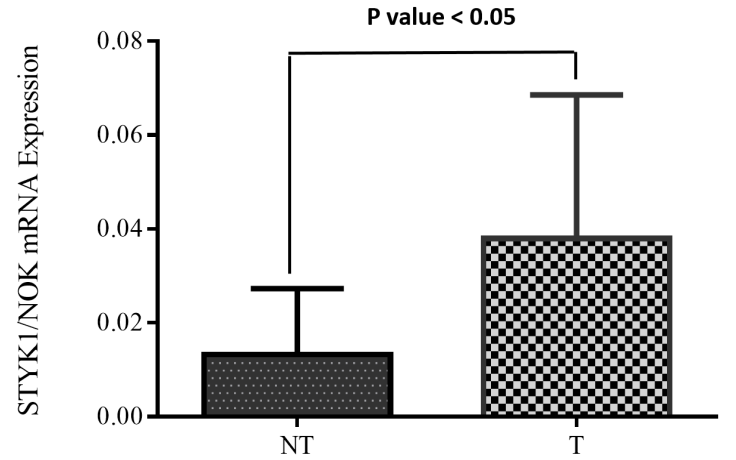

Figure 1. Expression of STYK1/NOK mRNA in Cancerous and Adjacent Non-Cancerous Tissues from CRC Patients. Expression of NOK mRNA was measured using quantitative RT-PCR and is reported relative to the amount of GAPDH mRNA. STYK1/NOK was differentially expressed between CRC tissues (T) and matched non-tumor adjacent tissues (NT). STYK1/NOK was significantly upregulated in CRC tissues compared to the matching adjacent non-tumor tissues $(\mathrm{P}<0.05$; paired t-test)

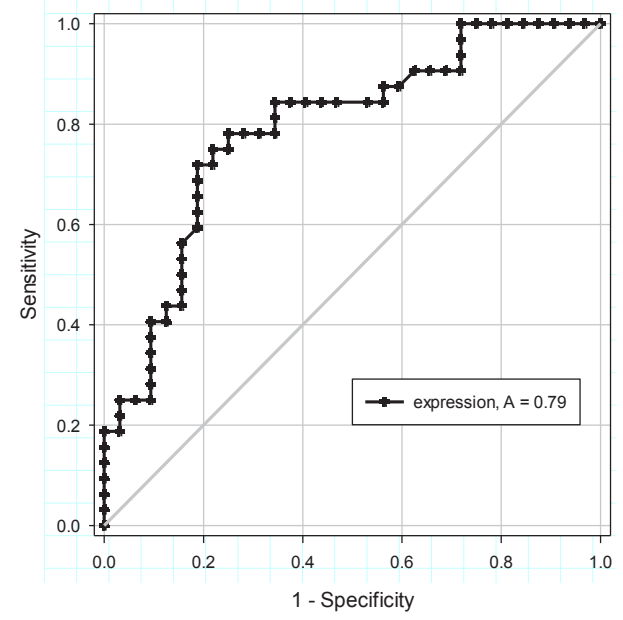

Figure 2. Receiver Operating Characteristic (ROC) Curve in Detection of CRC. The curve was automatically generated from 62 points of cutoff values set by the software SigmaPlot. The area under the ROC curve (AROC) is 0.79 out of 1

test from pre-test probability of 0.5 and cost ration of 1.00 . The optimal cut-off point was 0.198 with 0.78 and 0.75 Sensitivity and specificity respectively.

Correlation between STYK1/NOK expression levels and clinical staging in CRC patients. The relationship between STYK1/NOK expression levels and clinical stages determined by tumor size, invasion and metastasis to lymph nodes and distant organs was pursued in CRC samples. The results indicated that STYK1/NOK mRNA expression was elevated even at early stages of CRC and furthermore the median mean STYK1/NOK mRNA expression in stage I was $0.0375 \pm 0.0356$ compared with $0.0381 \pm 0.0244$ in other stages included II-IV ( $\mathrm{p}$ value $>0.05$ ) and all was shown in Figure 3.

Association between STYK1/NOK expression levels and other clinicopathological characteristics. In total, 


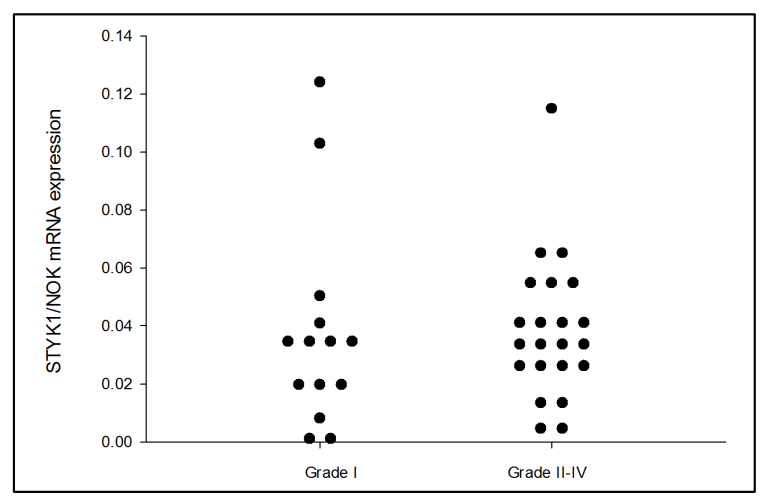

Figure 3.STYK1/NOK mRNA Expression at Different Clinical Stages of CRC. STYK1/NOK mRNA expression was measured using quantitative RT-PCR and expressed relative to the amount of GAPDH mRNA

Table 1. Relationships between NOK/STYK1 Expression Levels in Cancer Tissue Samples from Patients with CRC and Clinicopathological Features

\begin{tabular}{|c|c|c|c|c|}
\hline \multirow{2}{*}{$\begin{array}{l}\text { Feature } \\
\text { Gender }\end{array}$} & \multirow[t]{2}{*}{$\mathrm{N}$} & \multirow[t]{2}{*}{$\begin{array}{c}\mathrm{miR}-205 \\
\Delta \mathrm{Ct}\end{array}$} & \multicolumn{2}{|c|}{$\begin{array}{l}\text { Statistical p value } \\
\text { significance }\end{array}$} \\
\hline & & & & \\
\hline Male & 22 & $0.03 \pm 0.02$ & NS & 0.77 \\
\hline Female & 14 & $0.04 \pm 0.03$ & & \\
\hline \multicolumn{5}{|l|}{ Age (years) } \\
\hline$<65$ & 12 & $0.03 \pm 0.1$ & NS & 0.06 \\
\hline$\geq 65$ & 24 & $0.04 \pm 0.03$ & & \\
\hline \multicolumn{5}{|l|}{ Smoking status } \\
\hline Never & 11 & $0.07 \pm 0.01$ & NS & 0.15 \\
\hline Current or ex-smoker & 25 & $0.03 \pm 0.05$ & & \\
\hline \multicolumn{5}{|l|}{ Tumor location } \\
\hline Colon & 21 & $0.03 \pm 0.01$ & NS & 0.79 \\
\hline Rectum & 15 & $0.03 \pm 0.03$ & & \\
\hline \multicolumn{5}{|l|}{ Tumor size $(\mathrm{cm})$} \\
\hline$<5$ & 16 & $0.02 \pm 0.03$ & $* *$ & 0.0017 \\
\hline$\geq 5$ & 20 & $0.04 \pm 0.02$ & & 0.0017 \\
\hline \multicolumn{5}{|l|}{ Histological grade } \\
\hline Well differentiated & 17 & $0.03 \pm 0.02$ & NS & 0.93 \\
\hline Moderately differentiated & 13 & $0.04 \pm 0.03$ & & \\
\hline Poorly differentiated & 6 & $0.03 \pm 0.02$ & & \\
\hline \multicolumn{5}{|l|}{ pTNM stage } \\
\hline $\mathrm{I}+\mathrm{II}$ & 22 & $0.03 \pm 0.02$ & NS & 0.16 \\
\hline III+IV & 14 & $0.04 \pm 0.03$ & & \\
\hline \multicolumn{5}{|l|}{ Invasion into lymphatic vessels } \\
\hline Positive & 13 & $0.03 \pm 0.03$ & NS & 0.47 \\
\hline Negative & 23 & $0.03 \pm 0.03$ & & \\
\hline
\end{tabular}

*TNM, tumor-node-metastasis; Data presented as mean \pm SD; NS, not statistically significant $(\mathrm{P} \geq 0.05)$; $\mathrm{P}$-values obtained using Mann Whitney U test and Kruskal Wallis $\mathrm{H}$ test

36 patients ( 22 male, 14 female) were included in the study. The non-parametric test between the relative expression levels of STYK1/NOK in CRC cases and its clinicopathological characteristics have been exerted to investigate the associations with clinicopathological variables. Somewhat surprisingly, no significant associations were detected between expression of STYK1/ NOK and clinicopathological variables, including age, gender, tumor stage, differentiation, localization, smoking status and lymphocyte infiltration. However, increased expression levels of STYK1/NOK in patients with CRC tended to be associated with increased tumor sizes as shown by non-parametric tests ( $\mathrm{p}=0.0017$; Mann-Whitney $\mathrm{U}$ test). Patient characteristics with respect to increased STYK1/NOK expression are shown in Table 1.

\section{Discussion}

The past decades have seen advances in the diagnosis and treatment of colorectal cancer. However, due to the asymptomatic onset of colorectal cancer most patients are in advanced or metastatic condition at the time of diagnosis, resulting in a poor prognosis. RPTKs constitute the largest family of novel oncogenes and perturbation of RPTKs expression levels has been documented frequently in promoting cellular transformation and tumorigenesis (Blume-Jensen and Hunter, 2001, Danilkovitch-Miagkova and Zbar, 2002). These findings imply their importance in the development and/ or function of tumor cell as they function in such signal pathways that control cell shape, proliferation, differentiation and migration (Hirai et al., 1987, Berclaz et al., 1996, Robinson et al., 1996, Lamorte and Park, 2001). Existing diagnostic tools and biomarkers for CRC have many inherent deficiencies. Currently FGFR family is one of the most important group of RPTKs that their aberrant expression in CRC was frequently reported (Matsuda et al., 2012). However, it has been proved that FGFR is associated just with well-differentiated histological type (Sato et al., 2009). Furthermore the lack of correlation between FGFR evaluation and genetic/ mRNA level and its protein occurrence indicated that in order to exploit FGFR as a predictive marker, the assessment of the receptor at immunohistochemical level is required (Spano et al., 2005). STYK1/NOK is a naturally occurring from an unknown protein with certain sequence homology with FGFR/PDGFR family member and resembles the structural feature of FGFR4 missing complete extracellular domain (Liu et al., 2004). Several previous studies validated its dysregulation in acute Leukemia and breast, prostate, ovarian and lung cancer (Amachika et al., 2007, Kimbro et al., 2008, Chung et al., 2009, Jackson et al., 2009, Kondoh et al., 2009).

Using a QRT-PCR we have determined for first time, to our knowledge, STYK1/NOK was dramatically upregulated in CRC tissues, suggesting that high expression of STYK1/NOK involved in colorectal carcinogenesis. Its high sensitivity and specificity in optimal cut-off point confirmed its high diagnostic and prognostic value. We also assessed the association between expression elevation of STYK1/NOK and clinicopathological features of patients. However, in our cohort, just the increased tumor size in CRC was closely correlated with the high expression of STYK1/NOK. Although the increased tumor size in CRC was not part of the staging system, previous studies have suggested that tumor size is also an important prognostic factor in CRC (Kornprat et al., 2011). Thus, collectively, STYK1/NOK might be important biological marker in carcinogenesis of CRC and the high expression of STYK1/NOK may contribute to the proliferation of CRC tissues.

Specifically STYK1/NOK expression was found to be high even at the early clinical stages of CRC, which might explain why associations with other clinical variables were absent in our tumor panel. Nevertheless it is unambiguous whether the increased expression of STYK $1 / \mathrm{NOK}$ is one of the causes of CRC carcinogenesis or it is a consequence of normal colorectal cell transformation to tumor cells. 
In conclusion, our study showed that STYK1/NOK is dramatically upregulated in colorectal cancer tissues and that it could be used as a tumor marker. Furthermore we demonstrated that the high expression of STYK1/NOK is significantly associated with tumor size. Consistent with the observations reported by Liu et al. (Liu et al., 2004), which revealed the function of overexpressed STYK1/ NOK in tumorigenesis and metastasis of non-cancerous cells, and taking into account the structural differences between STYK1/NOK and RTKs, which lack extracellular domain, we speculate that over expression of STYK1/ NOK might result in growth factor-independent cell proliferation, surface adhesion-independent growth and may contribute to the carcinogenesis of CRC in synergism. The Confirmation of this theory requires large-scale and long-term follow-up studies. Further insights into the functional and clinical implications of STYK1/NOK may contribute to the early diagnosis of colorectal cancer and help with CRC therapeutic approaches.

\section{Acknowledgements}

Our gratitude goes to Tabriz Imam Reza Hospital nurses and staff at the endoscopy department and patients for their helpful collaboration. We would also like to thank doctors Kamal Boostani and Saleh Azadbakht for their kind help with the collection of the biopsy specimens.

\section{References}

Amachika T, Moriai R, Tsuji N, Watanabe N (2007). Diagnostic relevance of overexpressed mRNA of novel oncogene with kinase-domain (NOK) in lung cancers. Lung Cancer, 56, 337-40.

Barouni M, Larizadeh MH, Sabermahani A, Ghaderi H (2012). Markov's modeling for screening strategies for colorectal cancer. Asian Pac J Cancer Prev, 13, 5125-9.

Barrett J, Jiwa M, Rose P, Hamilton W (2006). Pathways to the diagnosis of colorectal cancer: an observational study in three UK cities. Fam Pract, 23, 15-9.

Berclaz G, Andres AC, Albrecht D, et al (1996). Expression of the receptor protein tyrosine kinase myk-1/htk in normal and malignant mammary epithelium. Biochem Biophys Res Commun, 226, 869-75.

Blume-Jensen P and Hunter T (2001). Oncogenic kinase signalling. Nature, 411, 355-65.

Chen Y, Li YH, Chen XP, et al (2005). Point mutation at single tyrosine residue of novel oncogene NOK abrogates tumorigenesis in nude mice. Cancer Res, 65, 10838-46.

Chung S, Tamura K, Furihata M, et al (2009). Overexpression of the potential kinase serine/ threonine/tyrosine kinase 1 (STYK 1) in castration-resistant prostate cancer. Cancer Sci, 100, 2109-14.

Danilkovitch-Miagkova A,Zbar B (2002). Dysregulation of Met receptor tyrosine kinase activity in invasive tumors. J Clin Invest, 109, 863-7.

Ding X, Jiang QB, Li R, Chen S, Zhang S (2012). NOK/ STYK1 has a strong tendency towards forming aggregates and colocalises with epidermal growth factor receptor in endosomes. Biochem Biophys Res Commun, 421, 468-73.

Fambrough D, McClure K, Kazlauskas A, Lander ES (1999). Diverse signaling pathways activated by growth factor receptors induce broadly overlapping, rather than independent, sets of genes. Cell, 97, 727-41.
Hirai H, Maru Y, Hagiwara K, Nishida J, Takaku F (1987). A novel putative tyrosine kinase receptor encoded by the eph gene. Science, 238, 1717-20.

Hubbard SR and Till JH (2000). Protein tyrosine kinase structure and function. Annu Rev Biochem, 69, 373-98.

Jackson KA, Handy J, Kimbro KS (2009). Aberrant STYK1 expression in ovarian cancer tissues and cell lines. J Ovarian Res, 2, 15-21.

Karimi K, Mahmoudi T, Karimi N, et al (2013). Is there an association between variants in candidate insulin pathway genes IGF-I, IGFBP-3, INSR, and IRS2 and risk of colorectal cancer in the Iranian population? Asian Pac J Cancer Prev, 14, 5011-6.

Kimbro KS, Willard M, Moore JA, Freeman S (2008). A novel gene STYK1/NOK is upregulated in estrogen receptor-alpha negative estrogen receptor-beta positive breast cancer cells following estrogen treatment. Mol Biol Rep, 35, 23-7.

Kondoh T, Kobayashi D, Tsuji N, Kuribayashi K, Watanabe N (2009). Overexpression of serine threonine tyrosine kinase $1 /$ novel oncogene with kinase domain mRNA in patients with acute leukemia. Exp Hematol, 37, 824-30.

Kornprat P, Pollheimer MJ, Lindtner RA, et al (2011). Value of tumor size as a prognostic variable in colorectal cancer: a critical reappraisal. Am J Clin Oncol, 34, 43-9.

Lamorte L and Park M (2001). The receptor tyrosine kinases: role in cancer progression. Surg Oncol Clin N Am, 10, 271-88,

Li YH, Wang YY, Zhong S, at al (2009). Transmembrane helix of novel oncogene with kinase-domain (NOK) influences its oligomerization and limits the activation of RAS/MAPK signaling. Mol Cells, 27, 39-45.

Liu L, Yu XZ, Li TS, et al (2004). A novel protein tyrosine kinase NOK that shares homology with platelet- derived growth factor/fibroblast growth factor receptors induces tumorigenesis and metastasis in nude mice. Cancer Res, 64, 3491-9.

Matsuda Y, Uenda J, Ishiwata T (2012). Fibroblast growth factor receptor 2: expression, roles, and potential as a novel molecular target for colorectal cancer. Patholog Res Int, 2012, 574768.

Robinson D, He F, Pretlow T, Kung HJ (1996). A tyrosine kinase profile of prostate carcinoma. Proc Natl Acad Sci USA, 93, 5958-62.

Safaee A, Moghimi-Dehkordi B, Fatemi SR, et al (2010). Characteristics of colorectal mucinous adenocarcinoma in Iran. Asian Pac J Cancer Prev, 11, 1373-5.

Samanian S, Mahjoubi F, Mahjoubi B, Mirzaee R, Azizi R (2011). MDR1 gene polymorphisms: possible association with its expression and clinicopathology characteristics in colorectal cancer patients. Asian Pac J Cancer Prev, 12, 3141-5.

Sato T, Oshima T, Yoshihara K, et al (2009). Overexpression of the fibroblast growth factor receptor- 1 gene correlates with liver metastasis in colorectal cancer. Oncol Re, 21, 211-6.

Shemirani AI, Haghighi MM, Zadeh SM, et al (2011). Simplified MSI marker panel for diagnosis of colorectal cancer. Asian Pac J Cancer Prev, 12, 2101-4.

Shepard HM, Brdlik CM, Schreiber H (2008). Signal integration: a framework for understanding the efficacy of therapeutics targeting the human EGFR family. J Clin Invest, 118, 3574-81.

Siegel R, Naishadham D, Jemal A (2013). Cancer statistics, 2013. CA Cancer J Clin, 63, 11-30.

Spano JP, Lagorce C, Atlan D, et al (2005). Impact of EGFR expression on colorectal cancer patient prognosis and survival. Ann Oncol, 16, 102-8.

Zwick E, Bange J, Ullrich A (2002). Receptor tyrosine kinases as targets for anticancer drugs. Trends Mol Med, 8, 17-23. 